\title{
Research on the Network Application of the Central Plain Martial Arts as an Intangible Cultural Heritage in the Internet + Era
}

\author{
Yin Tenglong \\ Public P. E. Department of Luoyang Normal University, Henan, Luoyang, 471022, P.R.China
}

13403792438@163.com

\begin{abstract}
Keywords: Internet+ Era; Central Plain martial arts; the intangible cultural heritage; networking; application

Abstract: The coming of the Internet + Era provides new chances and opportunities for protecting and inheriting Central plain martial arts as an intangible cultural heritage. The Central plain martial arts established the database of the intangible cultural heritage of Central plain martial arts by combining with the social characteristics of networking on market, service, technology and communication fully to achieve the digital technology development.Otherwise, they construct a full range of digital communication platform to realize networking applied in market. So that can protect Central Plain martial arts developing sustainably and healthily.
\end{abstract}

\section{The networking social characteristics in Internet + Era}

Internet + makes networking step in the age of 2.0. The whole society closelyrelies on the internet platformfrom production, transportation, service to information spreading, human daily consumption and so on. It demonstrates obvious networking social characteristics.

1.1 The networking of market

The networking of market is the trading mode that is based on the modern information technology and uses the internet as medium. It is characterized by discrete, non - centered, multi dimensional network structure and operation mode. The instant formation of information, instant transmission, real-time interaction and high - level human - machine interface constitute the form of transaction organization.Although the scope of networking market didn't change substantially, the trading modes of networking market changed totally. It means that "Online browsing, offline transactions" has changed into "Online browsing, online transactions". The modern market's network that was made by networking consumption market and networking service market has changed people's mind to traditional market.

\subsection{The networking of services}

In Internet + Era, the popularity of instant messaging programs makes communication between human beings more convenient. People build up their social circles based on the internet. In the Internet + Era, almost everyone has their own social tools like e-mail, we chat, weibo, QQ, Facebook, Twitter or anything else. Various services also base on these tools. Through the network platform, it makes travel booking, online shopping, information access, medical treatment and other kinds of services that is related to human's daily life more convenient, more hommization and more efficient.

1.3 The networking of technology

Technology is a way that people use the existing things to create new things or change the functions and performances of the existing things, such as raw materials, finished products, processes, tools, equipment, facilities, standards, specifications, indicators, measurement methods, etc. In the Internet + Era, the innovation and development of technology has been closely connected with internet. Those technical innovations, such as digitized technology, 3D printing technology, and cloud technology and so on, have epoch-making significances. Based on the global internet, the sharing and transmission of new technology is fast and accurate. People from all over the world are able to fully enjoy the achievements which are brought by technical progress.

1.4 The networking of dissemination

Internet + Era has become a global survival mode. Each industry achieves the corresponding information integration, processing and sharing, so that we can achieve rapid development. 
Network communication is developed rapidly in a short time. The speed, scale, areas that are impacted and forms of information that is spread by the internet far exceedthe media in the past. It greatly broadens people's horizon and enriches people's cultural life. Network communication has some strength, including great deal of information, fast dissemination, various ways of spreading. In addition, the process of communication becomes more and more interactive. The main body of audience can be reflected. The exchange is open and the main body of communication is wide.

\section{The Problems in the Inheritance of Intangible Cultural Heritage in Central Plain}

There are many kinds of Central Plain martial arts, which have a long history and far-reaching influence.Worldmartial arts observe Henan, Henan martial arts observe Shaolin,Central Plain martial arts are broad and profound. There are abundant resources, including hundreds of boxing camps and thousands of routines and skills. The skills are urgently to be excavated and organized which mostly scatter in folk. There are more than 40 kinds of boxing in the region of Zhongzhou which spread orderly and have complete system. Among them, Shaolin Quan, shadowboxing and other boxing is famous all over the world and through the country. Chang-style boxing, Mind LiuheQuan, YueshanBajiquan, Huang-style Chaquan, TongbeiQuan,MeihuaQuan, and other 25 kinds of boxing have been included in the national and provincial intangible cultural heritage. These show us that the martial arts as an intangible cultural heritage with deep culture are the valuable resources of Chinese traditional culture.

The development of traditional martial arts has always been concerned by academics, and it is also a pending and urgent proposition. With the developing and progress of time, many great changes have taken place on the land and environment that martial arts rely on. Those martial artists who make life by martial arts have been less and less. Martial arts have been a way of fitness.In the meantime, western sports were accepted by Chinese people gradually in modern times. The ways of fitness are of a great variety. The tradition of Wushu practicing has been marginalized gradually. The intangible cultural heritage of martial arts is non-renewable andtimeless. As time goes on, people who were carrying the heritage of martial arts and culture will eventually pass away. Those skills that are related with them will also disappear forever. It will cause irreparable regret for the integrity of Chinese culture. Protecting Central Plain martial arts cultural heritage is not only the need of understanding history, but also the need of creating new literature, new arts and new technology. The diversity of "Culture" makes a colorful world. Different schools of Central Plain martial arts, themselves, are the manifestation and embodiment of Chinese cultural diversity. And to a certain extent, protecting central Plain martial arts means to protect the diversity of Chinese culture.

\section{The networking application in inheriting of Central Plain martial arts}

3.1 The development of networking market ofCentral Plain martial arts

Different from material heritage, intangible cultural heritage realize its historical cognition value and meaning by the way of living transmission. Living transmission, live for the moment, is the most important characteristic of intangible cultural heritage. It is not rigescent and solid-stated, but living and developing. It needs people's participation to protect and inherit intangible cultural heritage in the environment of its production and development and develop in people's life processes. We should put the protection and inheriting of intangible cultural heritage in Central Plain in market. We should try to arouse the initiative and creative ability of martial arts heritage by market and make martial arts adapt to time development. Firstly, we need to regard the protection and inheriting of Central Plain martial arts as a sport industry and change the traditional thoughts that the protection of heritage relies on government. At present, Chinese sports industry is experiencing a historical golden time that "The country is doing. The government is promoting.The capital is flowing. The market is interacting." We should grasp this opportunity to find the way for living and developing in market. Secondly, we should release the resources of martial arts intangible cultural heritage actively and let enterprises participate in it. Make the martial arts heritage become 
the basic contents of enterprises' developing. Make resources function in culture, religion, health, fitness, tourism and other aspects. Only in this way, we can form a sports industry chain. Thirdly, we should break the concept of pedigree and area in inheriting of Central Plain martial arts. Based on the internet, we can enlarge each school's influence by accepting disciples' widely. At the same time, we need to increase the awareness of intellectual property protection. We should protect the rights of our schools' martial arts skills in spreading process by legal means.

3.2 Building the big data of Central Plain martial arts

Big data means a kind of data set that has a great data scale in the aspects of information access, storage, arrangement and analysis, rapid dataflow transformation, various date types and small valuable density. The meaning of big data technology is not in grasping huge data information, but in doing specialized processing to the meaningful data. Experiencing thousands of years developing, Central Plain martial arts have a great deal of schools and distribute widely. Except for those types of boxing that have been included in intangible cultural heritage list, the number of those skills that scatter in folk is huge. It's urgent to use the way of modern technology to protect these types of boxing like a "museum". And we also need to use the ways of texts, audios and videos to record them, inherit them and protect them.

The most straight and useful way to arrange the martial arts cultural heritage is to build a data base to realize the scientific storage, classification, retrieval and utilization of the intangible cultural heritage data. In Internet + Era, we can deepen human's acknowledge and understanding to martial arts which are intangible cultural heritages by the dissemination of mass media, internet and mobile terminal. The data base is a warehouse of organizing, storing and managing data. What's more, it is a process that makes the martial arts in Central Plain with thousands of years history date oriented and informationalized.

3.3The Digital Protection and Development Technology of Central Plain Martial Arts

The products of Central Plain martial arts which is an intangible cultural heritage need to use abundant texts, images, audios, videos and 3-D models to develop digital content products with the topic of intangible cultural heritage. The existing development of intangible cultural heritage includes two types, one is traditional media and the other is new media interactive form. The traditional media is mainly limited by the functions of traditional receiving terminal, for example, television and broadcast. Therefore, it develops some digital content products like television documentaries, animation films, books, audios and videos. By this way, the receiving terminal requirements are lower. But users often receive knowledge passively. It is not good for the studying and dissemination of intangible cultural heritage. With the development and universal of Multi touch screen, tablet PC, multimedia interactive TV these receiving terminals, digital products' forms have entered new media era. New media becomes the important carrier and means of upgrading and updating the technology of digital cultural industry. The biggest difference between new media and traditional ones is the interactive enhancement. It increases the initiative and interests of audience. Therefore, in the field of developing digital products, that using the new media digital products that are developed by those emerging information technology, such as virtual reality technology, human-computer interaction technology and stereoscopic display technology and so on, becomes popular. A new generation of somatosensory game machine takes Xbox as a representative. One of the martial arts fighting games broke through the limits of time and space. It displays dynamically, interactively the essence of martial arts. The digital products of intangible cultural heritage have become the development trend and mainstream by the three-dimensional, interactive and other new media's display form and the comprehensive utilization of the intangible cultural heritage. That is the direction of protecting and developing intangible cultural heritage in the future time.

3.4 Building up a Full Range of Disseminating Martial Arts Platform in Internet + Era

In Internet + Era, the dissemination and consumption of martial arts intangible cultural heritage has been combined as one. The technical system of digital dissemination and consumption use the spreading technology which includes satellite television, cloud technology, mobile terminal and so on to put the digital content products of martial arts intangible cultural heritage on the network, television, other media terminals and digital museums by showing the entities to spread and 
consume. Digital dissemination system of martial arts mainly involves Communications technology, dynamic scheduling technology, cloud computing technology, load balancing technology, multi-mode terminal seamless access technology based on triple play, multi terminal content adaptation decision technology, user oriented process management and quality evaluation technology, etc. For example, the stage show Shaolin Zen has a very high visibility at home and abroad. In the digital arena, they combined the real stage with digital products such as sound and light technology perfectly to make the audience in a reality that combined virtual martial arts environment with real actors, which gives the audience a sense of immersion.

\section{Acknowledgements}

Project: 2016 Soft Science Project of Science and Technology Department in Henan Province; Project name: A Research on the Development of Networking Technology and Market Application for "Living” Heritage of Central Plains Martial Arts as Intangible Cultural Heritage.

\section{References}

[1] Yu Zhijun. History of Chinese Traditional Martial Arts [M]. Beijing, China Renmin UniversityPress, 2006

[2] Intellectual Property Center of the Chinese Academy of Social Sciences.Study on the Protection of Intangible Cultural Heritage [M]. Beijing, Intellectual Property Publishing House, 2012:147

[3] Yu Dinghai, NiuAijun. Research on the Inheritance of Chinese Martial Arts on the Perspective of Intangible Cultural Heritage[M].Beijing, People's Sports Publishing House of China,2010:6-7

[4] Huang Yonglin, Tan Guoxin. Research on Digital Protection and Development of Intangible Cultural Heritage in China[J]. Central China Normal UniversityAcademic Journal(Humanities and Social Sciences), 2013, 51(2): 51-52 